\title{
VARIAÇÃO ANUAL DO RENDIMENTO E COMPOSIÇÃO QUÍMICA DOS COMPONENTES VOLÁTEIS DA Siparuna guianensis AUBLET
}

\author{
Carla Maria Abido Valentini* \\ Departamento de Química e Meio Ambiente, Instituto Federal de Educação, Ciência e Tecnologia de Mato Grosso, Campus Cuiabá \\ - Bela Vista, Av. Juliano Costa Marques, s/n, 78050-560 Cuiabá - MT, Brasil \\ Luiz Everson da Silva, Eduardo Nunes Maciel, Elton Franceschini, Paulo Teixeira de Sousa Jr. e Evandro Luiz Dall’’oglio \\ Departamento de Química, Universidade Federal de Mato Grosso, Av. Fernando Correa da Costa, s/n, 78060-900 Cuiabá - MT, Brasil \\ Maria de Fátima Barbosa Coelho \\ Departamento de Ciências Vegetais, Universidade Federal Rural do Semi-Árido, km 47 da BR-110 Costa e Silva, 59625-900 \\ Mossoró - RN, Brasil
}

Recebido em 18/10/09; aceito em 23/3/10; publicado na web em 20/7/10

\begin{abstract}
ANNUAL VARIATION OF YIELD AND CHEMICAL COMPOSITION OF VOLATILE COMPONENTS OF Siparuna guianensis Aublet. This work describes the volatile composition obtained by hidrodistillation of fresh leaves of Siparuna guianensis Aublet collected from Cuiabá (MT), Brazil. The composition of DCM extract of hydrolate was determined by GC-MS analysis and the results showed that the specie present a range of components according to their phenology and period of the leaves were collects. The highest volatile components yield was obtained during the reproductive period and the principal compound was the siparunone.
\end{abstract}

Keywords: Siparuna guianensis; volatile chemical composition; seasonal variability.

\section{INTRODUÇÃO}

Siparuna guianensis Aublet são arbustos monoicos pertencentes à família Siparunaceae, com folhas simples, alongadas a elípticas, membranáceas, de margens lisas, com flores unissexuais, e frutos deiscentes, encontrados desde a Nicarágua, por todo o norte da América do Sul até o Paraguai. ${ }^{1}$ Essa espécie é conhecida por vários nomes populares de acordo com o país e/ou região de sua distribuição no Brasil, em Mato Grosso é chamada de negramina. Os estudos realizados relataram o uso do decocto das folhas da espécie para serem usados principalmente na forma de banho tópico para sintomas de sinusite, febre, reumatismo, enxaqueca, gripe, dores no corpo e "malina", que é descrita como uma dor de cabeça causada pela exposição demasiada ao sol que provoca corrimento de sangue nasal. ${ }^{2}$

Estudos fitoquímicos da S. apiosyce, S. arianeae, S. dresslerana, S. gilgiana, S. nicaraguensis, S. pauciflora, S. tonduziana, S. patelliformis e $S$. macrotepala revelaram a presença de terpenos, alcaloides e flavonoides como constituintes majoritários. ${ }^{3} \mathrm{Na}$ S. guianensis, espécie estudada neste trabalho, autores revelaram a presença de terpenos, alcaloides e compostos não terpênicos.

A influência das condições climáticas na composição da composição volátil da $S$. guianensis foi observada por detecção de ampla variação dos componentes da composição volátil obtida de plantas coletadas no estado de Rondônia e na Zona da Mata de Minas Gerais durante diferentes meses do ano. Fischer et al. estudaram os componentes voláteis das folhas e frutos dessa espécie coletados no sudoeste do cerrado brasileiro e, comparando seus resultados com estudos de duas regiões da Amazônia, e uma do Panamá, inferiram que, no cerrado, os principais constituintes das folhas da $S$. guianensis seriam as metilcetonas e ácidos graxos, enquanto que na região norte, os componentes majoritários seriam os sesquiterpenos. ${ }^{4}$

Como a época em que uma espécie é coletada, associada a uma série de fatores inerentes aos locais onde a espécie se encontra é um dos fatores de maior importância no conteúdo e na quantidade da com-

\footnotetext{
*e-mail: carla.valentini@blv.ifmt.edu.br
}

posição volátil,${ }^{5}$ o objetivo deste estudo foi quantificar e identificar os principais compostos químicos da composição volátil extraídos de folhas de Siparuna guianensis numa área de Cerrado em Mato Grosso, durante 1 ano, para averiguar a sua possível variação em função da fenologia da espécie e das variações climáticas do local.

\section{PARTE EXPERIMENTAL}

\section{Material vegetal}

As folhas da Siparuna guianensis foram coletadas mensalmente, entre 8:00 e 9:00 h, de 20 indivíduos georreferenciados para análise fenológica no Bosque Paulo Siqueira (15³3’33,81”S e 56 03’34,30”O), no município de Cuiabá - MT, de novembro de 2007 a outubro de 2008. A amostra composta coletada foi acondicionada em saco plástico e, logo após, transportada ao Laboratório de Pesquisa em Química de Produtos Naturais na UFMT. A identificação da espécie foi realizada no Herbário da UFMT, onde se encontram depositadas as exsicatas sob os registros 38354 e 38356.

\section{Levantamento dos dados meteorológicos e fenológicos}

Os dados meteorológicos de temperatura, umidade relativa do ar e índice pluviométrico foram cedidos pelo INPE (Instituto Nacional de Pesquisas Espaciais), de uma estação localizada nas coordenadas 15³3'18,69' S e 5604'09,69' O, a 1,4 km do Bosque Paulo Siqueira, e a intensidade dos eventos fenológicos foi estimada para cada indivíduo seguindo-se os critérios de Fournier. ${ }^{6}$

\section{Extração dos componentes voláteis}

O material vegetal, composto de folhas frescas sadias, cortadas em frações de aproximadamente $1 \mathrm{~cm}$, foram submetidas à hidrodestilação durante $3 \mathrm{~h}$, com extrator do tipo Clevenger. ${ }^{7} \mathrm{O}$ hidrolato foi particionado em funil de separação utilizando-se diclorometano (DCM) e as frações orgânicas obtidas foram reunidas e secas com 
cloreto de cálcio anidro $\left(\mathrm{CaCl}_{2}\right)$. O sal foi removido por filtração a vácuo e o solvente evaporado à temperatura ambiente.

\section{Composição química dos componentes voláteis}

Os principais componentes da fração volátil foram identificados por cromatografia gasosa acoplada ao espectrômetro de massas (CGEM), equipamento Shimadzu (QP 5050A) quadrupolo operando a 70 eV. As análises cromatográficas foram efetuadas usando-se coluna capilar de sílica fundida tipo DB-5 (30 m de comprimento; 0,25 mm de diâmetro interno; $0,25 \mu \mathrm{m}$ de espessura do filme). As temperaturas do forno e detector foram de 110 e $340{ }^{\circ} \mathrm{C}$, respectivamente, e a temperatura da interface de $320^{\circ} \mathrm{C}$. O forno foi programado para efetuar o seguinte gradiente: $110-140{ }^{\circ} \mathrm{C}$ a $5{ }^{\circ} \mathrm{C} / \mathrm{min}$, de $140-290$ a $20{ }^{\circ} \mathrm{C} / \mathrm{min}$ e de $290-330$ a $5{ }^{\circ} \mathrm{C} / \mathrm{min}$. O gás hélio foi empregado como gás de arraste, com fluxo de $1,2 \mathrm{~mL} / \mathrm{min}$, e o tempo total de corrida foi de $35 \mathrm{~min}$.

A identificação dos componentes foi baseada na comparação de seus espectros de massas com os disponíveis na base de dados da biblioteca Wiley (software class 5k) do equipamento, por comparação visual com dados da literatura e confirmada pela determinação de seus índices de Kováts, que foram calculados relativamente aos tempos de retenção de uma série de n-alcanos. ${ }^{8}$

\section{RESULTADOS E DISCUSSÃO}

\section{Rendimento da composição volátil em função da fenologia e das variáveis meteorológicas}

O clima da região é do tipo Aw segundo a classificação de Köppen, ou seja, tropical semiúmido, com temperatura média de 24 a $26^{\circ} \mathrm{C}$, com 4 a 5 meses secos e duas estações bem definidas: uma seca (outono-inverno) e uma chuvosa (primavera-verão), com índice pluviométrico anual de 1250 a $1500 \mathrm{~mm} .^{9}$

Na Figura 1 são apresentadas a variação meteorológica do local e as fenofases da S. guianensis durante o ano de coleta e, na Tabela 1, os rendimentos obtidos dos principais componentes da composição volátil.

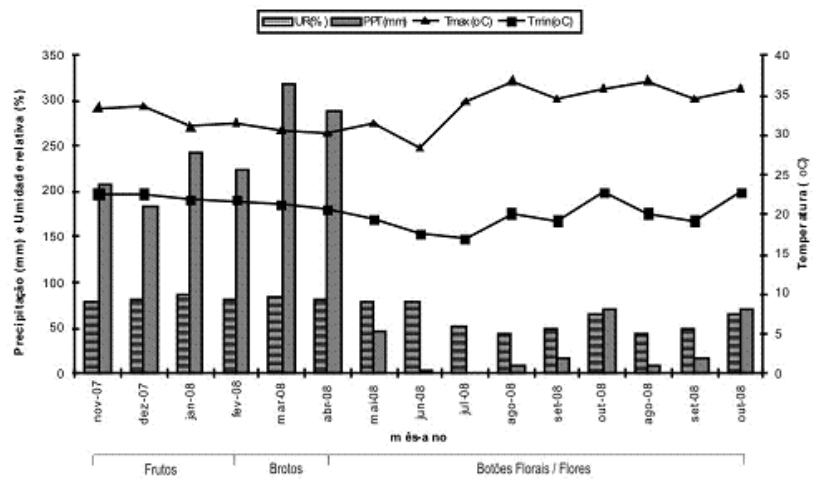

Figura 1. Variação meteorológica local ao longo dos meses de coleta

Observou-se que a maior quantidade da composição volátil da $S$. guianensis ocorreu nos períodos reprodutivos, quando a planta estava emitindo botões florais, flores e frutos, enquanto que no período vegetativo esta ocorreu em menor quantidade. Esses dados sugerem que nos períodos de frutificação e floração a produção da composição volátil é intensificada.

O melhor rendimento da composição volátil ocorreu no mês de julho de 2008 que, dentre os meses avaliados, foi o mais seco e com maior número de flores abertas. A precipitação anual exerce um efeito sobre a vegetação, influenciando diretamente a produção dos compostos voláteis. Doran explicou que além desses compostos
Tabela 1. Rendimento mensal da composição volátil das folhas da S. guianensis

\begin{tabular}{lccc}
\hline Mês/ano & $\begin{array}{c}\text { Massa da folha } \\
\text { utilizada }(\mathrm{g})\end{array}$ & $\begin{array}{c}\text { Massa da composição } \\
\text { volátil obtida }(\mathrm{g})\end{array}$ & $\begin{array}{c}\text { Rendimento } \\
(\%)\end{array}$ \\
\hline Nov-07 & 53,800 & 0,060 & 0,112 \\
Dez-07 & 31,500 & 0,106 & 0,336 \\
Jan-08 & 58,690 & 0,126 & 0,215 \\
Fev-08 & 33,050 & 0,033 & 0,100 \\
Mar-08 & 52,040 & 0,052 & 0,100 \\
Abr-08 & 43,150 & 0,108 & 0,250 \\
Mai-08 & 45,740 & 0,138 & 0,302 \\
Jun-08 & 36,380 & 0,173 & 0,476 \\
Jul-08 & 33,480 & 0,205 & 0,612 \\
Ago-08 & 42,330 & 0,179 & 0,423 \\
Set-08 & 36,480 & 0,074 & 0,203 \\
Out-08 & 28,230 & 0,058 & 0,205 \\
\hline
\end{tabular}

poderem estar envolvidos nas interações animal-planta, planta-microorganismos e planta-planta, com a finalidade de sobrevivência e/ou manutenção da planta, conferindo a ela capacidade de adaptação às condições do meio em que vive, podem apresentar funções ecológicas como, por exemplo, nesse caso da estiagem, a redução da perda de água. Andrade e Gomes observaram que folhas maduras de $E$. citriodora coletadas no outono (período de estiagem) registraram maiores rendimentos de óleo essencial do que as coletada no verão (período chuvoso). Pitarevic postulou que um longo período de seca em populações naturais de Salvia officinalis foi a base para uma produção elevada de óleo essencial. Com Artemisia annua, Gupta et al. obtiveram maior porcentagem de óleo essencial a partir de plantas em floração. Vitti e Brito relataram maior rendimento de óleo essencial com folhas de eucalipto nos meses mais secos do ano (abril a setembro) e concluíram que quanto menor o teor de umidade nas folhas, melhor a qualidade da essência. ${ }^{10}$

Catellani et al. ${ }^{4}$ avaliando a mesma espécie do presente estudo, $S$. guianensis, coletada em Viçosa-MG, observaram que no outono foram obtidos os maiores valores do teor de óleo essencial, ressaltando que nesse mês, para aquela região, ocorreu o menor índice de pluviosidade do ano pesquisado, e que nessa estação a espécie começou a emitir botões florais. Também observaram que na primavera, fase de frutificação e brotação, ocorreram os menores valores de óleo essencial. Explicaram esse fato justificando que neste período pode haver limitações de recursos, considerando que a produção de óleos essenciais envolve gasto de energia e poderia competir com esses drenos, que estão relacionados com o crescimento e a sobrevivência do vegetal.

\section{Identificação dos compostos da composição volátil}

Em nível de variabilidade das classes de compostos em relação à composição volátil, 70\% dos compostos identificados são sesquiterpenos.

Os monoterpenos oxigenados ocorreram apenas nos meses de fevereiro, março e setembro de 2008, sendo que sua produção foi intensificada no final do período chuvoso, que coincidiu com o período de brotação da espécie.

Os sesquiterpenos hidrocarbonetos apareceram no período chuvoso e de transição chuvoso-seco, com maior produção em fevereiro de 2008, onde se observou o final da frutificação e início da brotação da $S$. guianensis.

Os sesquiterpenos oxigenados ocorreram e predominaram em todos os meses observados, sendo que em abril de 2008, foi identificada sua maior produção, período de transição chuvoso-seco e de intensa brotação da espécie. 
Os compostos não terpênicos só não foram identificados em fevereiro de 2008, mês de maior produção dos sesquiterpenos hidrocarbonetos. Sua maior quantidade ocorreu em agosto de 2008, período em que houve diminuição dos sesquiterpenos oxigenados. Este mês é muito seco no local, e a espécie apresentava botões florais e flores. É possível que os compostos não terpênicos sejam os principais responsáveis pela proteção das folhas da S. guianensis contra a perda de água no cerrado de Mato Grosso.

Na Figura 2 são apresentadas as fórmulas estruturais dos compostos identificados na composição volátil das folhas da S. guianensis e nas Tabelas 2 e 3, as quantidades mensais de cada composto.
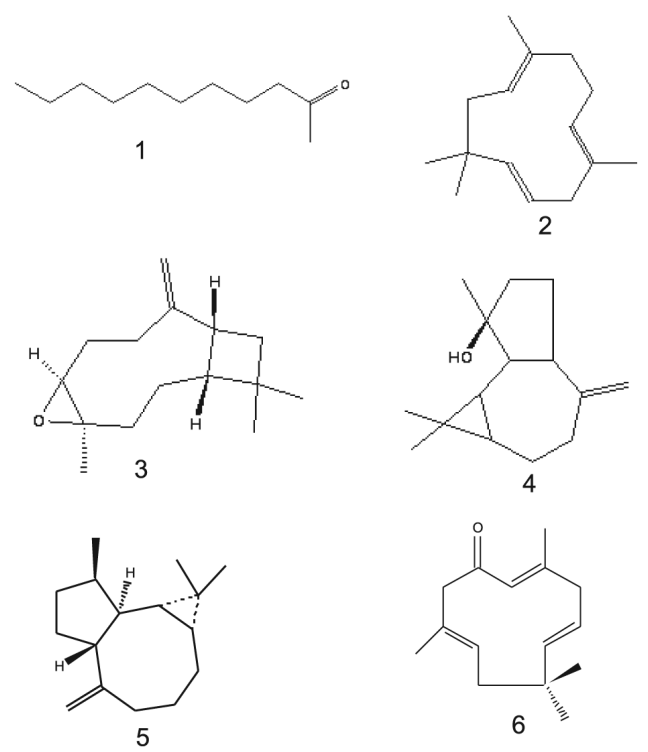<smiles>C=C(C)OC1CC(C(=C)C)CCC1C</smiles><smiles>CC1(C2CC[C@]3(C)O[C@@H]3C2)CO1</smiles>

8
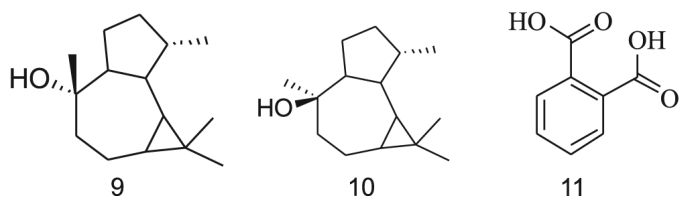

Figura 2. Compostos identificados na composição volátil das folhas da $S$. guianensis (1. 2-undecanona, 2. humuleno, 3. óxido de cariofileno, 4. espatulenol, 5. aromadendreno, 6. siparunona, 7. acetato de di-hidrocarvil, 8. dióxido de limoneno, 9. ledol, 10. viridiflorol, 11. ácido 1,2-benzenodioico)

Exceto para a amostra do mês de novembro de 2007, nos demais meses analisados, a siparunona foi o componente majoritário, com média mensal igual a 41,64 $\pm 12,68$ (DP), e com maior produção no mês de junho de 2008, época seca, com as menores temperaturas máxima e mínima. O ledol foi identificado nas amostras de todos os meses, e o espatulenol só não foi identificado no mês de agosto de 2008, sendo que os mesmos foram identificados em maior quantidade na época de transição seca-chuvosa. Esses compostos identificados em praticamente todas as amostras mensais (siparunona, ledol e espatulenol) tiveram um menor rendimento nos meses chuvosos. $\mathrm{O}$ óxido de cariofileno, o ácido 1,2-benzenodioico e o acetato de di-hidrocarvil tiveram sua maior produção na época seca (agosto de 2008), onde se registrou a maior média da temperatura máxima do ano. Os dois
Tabela 2. Quantidades mensais dos compostos identificados na composição volátil das folhas de $S$. guianensis coletadas no Cerrado de Mato Grosso nos meses de novembro de 2007 a março de 2008 e seus tempos de retenção (IR/ min) e índices de Kováts (IK)

\begin{tabular}{|c|c|c|c|c|c|c|c|c|}
\hline \multicolumn{2}{|r|}{ Compostos } & \multirow{2}{*}{$\begin{array}{c}\mathrm{TR} / \mathrm{min} \\
7,34\end{array}$} & \multirow{2}{*}{$\frac{\mathrm{IK}}{1293}$} & \multicolumn{4}{|c|}{ nov/07 dez/07 jan/08 fev/08 } & \multirow{2}{*}{$\frac{\operatorname{mar} / 08}{-}$} \\
\hline 1 & 2-undecanona & & & 0,58 & - & 0,44 & - & \\
\hline 2 & Humuleno & 7,84 & 1455 & - & - & 2,16 & 4,72 & - \\
\hline 3 & Óxido cariofileno & 8,27 & 1583 & 1,92 & - & 1,65 & - & 1,25 \\
\hline 4 & Espatulenol & 8,50 & 1574 & 10,8 & 10,22 & 2,8 & 9,09 & 6,26 \\
\hline 5 & Aromadendreno & 8,81 & 1440 & - & - & - & 0,51 & 1,15 \\
\hline 6 & Siparunona & 9,78 & 1663 & 21,09 & 52,54 & 32,34 & 43,76 & 54,47 \\
\hline 7 & $\begin{array}{c}\text { Acetato de } \\
\text { di-hidrocarvil }\end{array}$ & 10,18 & 1306 & - & - & - & - & - \\
\hline 8 & Dióxido de limoneno & 10,53 & 1137 & - & - & - & 1,74 & 10,81 \\
\hline 9 & Ledol & 10,70 & 1562 & 6,94 & 1,18 & 10,24 & 1,22 & 6,88 \\
\hline 10 & Viridiflorol & 10,81 & 1595 & 26,47 & 13,77 & - & - & 0,56 \\
\hline 11 & $\begin{array}{c}\text { Ácido } \\
\text { 1,2-benzenodioico }\end{array}$ & 13,08 & 1927 & - & 3,97 & - & - & 0,31 \\
\hline & $\begin{array}{c}\text { Monoterpenos } \\
\text { oxigenados }\end{array}$ & & & - & - & - & 1,74 & 10,81 \\
\hline & $\begin{array}{l}\text { Sesquiterpenos } \\
\text { hidrocarbonetos }\end{array}$ & & & - & - & 2,16 & 5,23 & 1,15 \\
\hline & $\begin{array}{c}\text { Sesquiterpenos } \\
\text { oxigenados }\end{array}$ & & & 67,22 & 77,71 & 47,03 & 54,07 & 69,42 \\
\hline & $\begin{array}{l}\text { Compostos não } \\
\text { terpênicos }\end{array}$ & & & 0,58 & 3,97 & 0,44 & - & 0,31 \\
\hline & Total & & & 67,8 & 81,68 & 49,63 & 61,04 & 81,69 \\
\hline
\end{tabular}

Tabela 3. Quantidades mensais dos compostos identificados na composição volátil das folhas de $S$. guianensis coletadas no Cerrado de Mato Grosso nos meses de abril de 2008 a outubro de 2008

\begin{tabular}{|c|c|c|c|c|c|c|c|c|}
\hline \multirow[b]{2}{*}{1} & \multirow{2}{*}{$\frac{\text { Compostos }}{\text { 2-undecanona }}$} & \multicolumn{3}{|c|}{ abr/08 mai/08 jun/08 } & \multicolumn{3}{|c|}{ jul/08 ago/08 set/08 } & \multirow{2}{*}{$\frac{\text { out/08 }}{-}$} \\
\hline & & 1,05 & 4,92 & 0,9 & - & - & - & \\
\hline 2 & Humuleno & - & - & - & - & - & - & - \\
\hline 3 & Óxido cariofileno & - & 3,15 & - & 2,62 & 19,44 & - & 2,25 \\
\hline 4 & Espatulenol & 6,8 & 10,03 & 3,53 & 3,6 & - & 3,7 & 17,21 \\
\hline 5 & Aromadendreno & & - & 4,56 & - & - & - & - \\
\hline 6 & Siparunona & 59,5 & 30,17 & 59,77 & 40,91 & 32,59 & 41,34 & 31,23 \\
\hline 7 & $\begin{array}{l}\text { Acetato de di- } \\
\text { hidrocarvil }\end{array}$ & - & - & - & 22,16 & 23,89 & 1,35 & 5,4 \\
\hline 8 & Dióxido de limoneno & - & - & - & - & - & 2,45 & - \\
\hline 9 & Ledol & 7,14 & 6,19 & 9,41 & 6,05 & 6,27 & 10,49 & 4,85 \\
\hline 10 & Viridiflorol & 7,2 & 12,06 & - & 3,16 & - & - & 5,15 \\
\hline \multirow[t]{6}{*}{11} & $\begin{array}{l}\text { Ácido1,2- } \\
\text { benzenodioico }\end{array}$ & - & - & 1,01 & 1,62 & 5,87 & 0,75 & - \\
\hline & $\begin{array}{l}\text { Monoterpenos } \\
\text { oxigenados }\end{array}$ & - & - & - & - & - & 2,45 & - \\
\hline & $\begin{array}{l}\text { Sesquiterpenos } \\
\text { hidrocarbonetos }\end{array}$ & - & - & 4,56 & - & - & - & - \\
\hline & $\begin{array}{l}\text { Sesquiterpenos } \\
\text { oxigenados }\end{array}$ & 80,64 & 61,6 & 72,71 & 56,34 & 58,3 & 55,53 & 60,69 \\
\hline & $\begin{array}{l}\text { Compostos não } \\
\text { terpênicos }\end{array}$ & 1,05 & 4,92 & 1,91 & 23,78 & 29,76 & 2,1 & 5,4 \\
\hline & Total & 81,69 & 66,52 & 79,18 & 80,12 & 88,06 & 60,08 & 66,09 \\
\hline
\end{tabular}

primeiros compostos tiveram sua menor produção no mês em que ocorreu o maior índice de precipitação acumulado do ano (março de 2008), e o último na transição seca-chuvosa. Já a 2-undecanona teve sua maior produção na transição chuvosa-seca, e sua menor produção no período chuvoso (janeiro de 2008). 
Em dois estudos realizados em Rio Branco - AC foram encontrados compostos majoritários diferentes para as folhas de S. guianensis: siparunona e espatulenol. Vale ressaltar que no trabalho de Rebouças não foi encontrado o espatulenol. ${ }^{4}$

$\mathrm{O}$ viridiflorol, que teve identificação nas amostras das épocas chuvosa e transição chuvosa-seca, foi encontrado como composto majoritário da Melaleuca quinquenervia e foi associado a plantas de baixa palatabilidade dos herbívoros. ${ }^{11}$

O dióxido de limoneno e o humuleno, que são encontrados na maioria das plantas aromáticas, ${ }^{12}$ só foram identificados nos meses chuvosos e, ao contrário dos demais compostos, tiveram sua maior produção no mês mais chuvoso avaliado (março de 2008). No estudo com Leptospermun madidum ssp savitum e Leptospermun flavescens Magalhães ${ }^{13}$ observou que a produção do humuleno foi maior na estação chuvosa do que na estação seca.

O composto 2-undecanona, identificado na composição volátil de alguns dos meses do presente estudo, mas em quantidades bem inferiores ao composto majoritário, diferiu do trabalho de Fischer et al. ${ }^{4}$ que o identificaram como principal composto em folhas de S. guianensis coletadas no mês de outubro de 2000 em Mogi das Cruzes - SP, Cerrado do Sudoeste do Brasil. Vale ressaltar que, em outubro de 2008, a substância 2-undecanona não foi identificada nas folhas que foram coletadas neste trabalho no Cerrado de Mato Grosso.

\section{CONCLUSÕES}

A espécie Siparuna guianensis apresenta em suas folhas variação do rendimento da composição volátil em função da sua fenologia e variações meteorológicas locais, sendo obtido melhor rendimento no período reprodutivo. Houve predominância de sesquiterpenos e o composto majoritário para os meses avaliados foi a siparunona, identificado apenas no estudo de Rebouças, ${ }^{4}$ na região Amazônica. Comparando-se com outros estudos pode-se inferir que há variação na composição química dos componentes voláteis dependendo do local de coleta da amostra, porém não é possível sugerir quais compostos seriam possíveis de serem encontrados em determinada região, pois há carência de informações na literatura que poderiam enriquecer a discussão, como época de coleta do material, fenologia da espécie nos momentos da coleta e dados meteorológicos dos locais. Portanto, para o local estudado, com o objetivo de um maior rendimento da composição volátil, devem-se evitar coletas de folhas no período vegetativo, que para o Cerrado de Mato Grosso compreende os meses de fevereiro e março.

\section{REFERÊNCIAS}

1. Renner, S. S.; Hausner, G.; Monograph of Siparunaceae. Flora Neotropica 95, The New York Botanical Garden Press: New York, 2005.
2. Milliken, W.; Albert, B.; Econ. Bot. 1996, 50, 10; Bourdy, G.; Dewalt, S. J.; Chávez de Michel, L. R.; Roca, A.; Deharo, E.; Muñoz, V.; Balderrama, L.; Quenevo, C.; Gimenez, A.; J. Ethnopharmacol. 2000, 70, 87; Rodrigues, V. E. G.; Carvalho, D. A.; Ciênc. Agrotec. 2001, 25, 102; Lorenzi, H.; Matos, F. J. A.; Plantas medicinais no Brasil: nativas e exóticas, $4^{\mathrm{a}}$ ed., Instituto Plantarum: Nova Odessa, 2002; Souza, C. D.; Felfili, J. M.; Acta Bot. Bras. 2006, 20, 135; Valentini, C. M. A; Rodríguez-Ortíz, C. E.; Coelho, M. F. B; Rev. Bras. Pl. Med. 2010, 12, 96.

3. Fischer, D. C. H.; Gonçalves, M. I.; Oliveira, F.; Alvarenga, M. A.; Fitoterapia 1999, 70, 322; Leitão, G. G.; Simas, N. K.; Soares, S. S.V.; Brito, A. P. P.; Claros, B. M. G.; Brito, T. B. M.; Monache, F. D.; J. Ethnopharmacol. 1999, 65, 87; Lopez, J. A.; Laurito, J. G.; Lin, F.; Duah, F. K.; Sharaf, M.; Aly, Y.; Wong, L. K.; Shiff Jr, P. L.; Planta Med. 1990, 56, 492; Gerard, R. V.; MacLean, D. B.; Antonio, T. M.; Phytochemistry 1986, 25, 2155; El-Seedi, H.; Ghia, F.; Torssell, K. B. G.; Phytochemistry 1994, 35, 1495.

4. Antônio, T. M.; Waller, G. R.; Mussinan, C. J.; Chem. Ind. 1984, 16, 514; Rebouças, L. M. C.; Dissertação de Mestrado, Universidade Federal do Ceará, Brasil, 1984; Machado, S. M. F.; Facundo, V. A.; Militão, J. S. L. T.; Morais, S. M.; Machado, M. I. L.; Ribeiro, A. S.; J. Essent. Oil Res. 1998, 10, 708; Zoghbi, M. G. B.; Andrade, E. H. A.; Santos, A. S.; Silva, M. H. L; Maia, J. G. S; Silva, M. H. L.; Haia, J. G. S.; J. Essent. Oil Res. 1998, 10, 543; Viana, F. A.; Andrade-Neto, M.; Poliquen, Y. B. M.; Uchoa, D. E. A.; J. Essent. Oil Res. 2002, 14, 60; Braz-Filho, R.; Gabriel, S. J.; Gottlieb, O. R.; Bichara, M. G. A. E.; Maia, J. G. S.; Phytochemistry 1976, 15, 1187; Fischer, D. C. H.; Limberger, R. P.; Henriques, A. T.; Moreno, P. R .H.; J. Essent. Oil Res. 2005, 17, 101; Castellani, D. C.; Casali, V. W. D.; Souza, A. L.; Cecon, P. R.; Cardoso, C. A.; Marques, V. B.; Rev. Bras. Pl. Med. 2006, 8, 62.

5. Gobbo-Neto, L.; Lopes, N. P.; Quim. Nova 2007, 30, 374.

6. Fournier, L. A.; Turrialba 1974, 24, 422.

7. Farmacopeia Brasileira, $4^{\mathrm{a}}$ ed., Atheneu: São Paulo, 2000

8. Adams, R. P.; Identification of essential oils components by gas chromatography/Quadrupole mass spectroscopy, Allured: Carol Stream, 2001.

9. Maitelli, G. T.; Revista Mato-grossense de Geografia 1997, 1, 150.

10. Evans, W. C.; Farmacognosia, $13^{\text {th }}$ ed., Nueva Editorial Interamericana: México, 1991; Doran, J. C. Em Eucalyptus leaf oils: use, chemistry, distillation and marketing; Boland, D. J.; Brophy, J. J.; House, A. P. N., eds.; Inkata: Melbourne 1991; Andrade, A. M.; Gomes, S. S.; Floresta Ambient. 2000, 7, 181; Pitarevic, I.; J. Nat. Prod. 1984, 47, 409; Gupta, S. K.; Singh, P.; Bajpai, P.; Ram, G.; Digvijai, S.; Gupta, M. M.; Jaim, D. C.; Khanuja, S. P. S.; Kumar, S.; Ind. Crop. Prod. 2002, 16, 2217; Vitti, A. M. S.; Brito, J. O.; Sci. Florestalis 1999, 56, 145.

11. Silva, C. J.; Barbosa, L. C. A.; Maltha, C. R. A.; Pinheiro, A. L.; Ismail, F. M. D.; Flav. Frag. J. 2007, 22, 474.

12. Almeida, L. R. F.; Delachiave, M. E. A.; Marques, M. O. M.; Rev. Bras. Pl. Med. 2005, 8, 35 .

13. Magalhães, C. G.; Barbosa, L. C. A.; Maltha, C. R. A.; Pinheiro, A. L.; Demuner, A. J.; Resumos da $48^{\circ}$ Congresso Brasileiro de Química, Rio de Janeiro, Brasil, 2008. 